\title{
Hommage à Gérard Mégie
}

Les 12 et 13 janvier 2006, ont eu lieu, dans la grande salle de 1'Unesco à Paris, des « Journées Gérard Mégie». Elles ont été l'occasion d'un hommage exceptionnel à l'homme autant qu'au scientifique. Le lieu de cette rencontre et la liste des organismes qui se sont associés pour la patronner (Académie des sciences, ministère délégué à l'Enseignement supérieur et à la Recherche, CNRS, Institut universitaire de France, Université Pierre et Marie Curie, Université de Versailles Saint-Quentinen-Yvelines, CNES, INSU, Institut Pierre-Simon Laplace [IPSL], Mouvement universel de la responsabilité scientifique) sont déjà, à eux seuls, édifiants quant à l'audience dont il bénéficiait. Les différentes facettes de sa vie professionnelle ont été tour à tour évoquées à travers des témoignages souvent empreints d'émotion. Ce parcours a mis en évidence l'intensité de l'activité et la richesse de la personnalité de Gérard Mégie. Une personnalité qui s'avère bien, à l'issue de ces deux journées, hors pair.

Les mêmes mots sont sans cesse revenus sur les lèvres des témoins pour parler de ses qualités en tant que personne : courtoisie, générosité, droiture, écoute, respect de l'autre, sens des responsabilités, engagement, ouverture d'esprit...

Tant de tâches assumées simultanément! Tant de combats - car c'est bien de combats qu'il s'agit, que ce soit pour bâtir ou pour convaincre - menés tous azimuts! Et, dans le même temps, une telle disponibilité, en apparence sans limites! C'est là la grâce que donne, face au temps, la vivacité de l'intelligence. Cette vivacité que l'on ressentait pleinement et dont on se nourrissait quand on était face à Gérard Mégie.

Dans son activité d'enseignant, à ses qualités de pédagogue s'ajoutaient celles de la relation pleine de tact et soucieuse d'accompagner qu'il instaurait avec ses étudiants. Dans son activité scientifique, il s'est avéré un animateur, un rassembleur, un bâtisseur dynamique et efficace et un grand innovateur, que ce soit au niveau de son laboratoire ou de sa discipline ou, dans la coopération scientifique, au niveau international aussi bien qu'européen. Il lui aura fallu treize années d'efforts et de rares qualités de négociateur pour qu'enfin l'IPSL sorte de terre! «Gérard Mégie, c'est celui qui se fait un point d'honneur de rendre possible...", a dit l'un des intervenants.

Sa carrière scientifique fut brillante et internationalement reconnue. Pionnier dans son domaine de compétence avec, entre autres, la mise au point du LIDAR, instrument conçu pour analyser la composition de la haute atmosphère, il le fut également dans la réflexion sur la démarche scientifique, sur l'organisation de la recherche, sur les rapports sciences/sociétés. Ce qui s'exprima tout particulièrement dans son adhésion franche et nette à l'idée que l'interdisciplinarité était devenue une nécessité. Cette conviction avait chez lui trois fondements.

Elle découlait tout d'abord logiquement de la question de recherche à laquelle il avait choisi de consacrer ses travaux, celle de l'évolution de la couche d'ozone dans la stratosphère et de la teneur en ozone dans la troposphère : déjà en elle-même au carrefour de la chimie et de la physique, cette question conduit en outre à s'interroger sur les activités humaines qui sont impliquées dans cette évolution et sur la façon dont les connaissances scientifiques peuvent entrer en composition avec les contraintes économiques et sociales pour aller vers des solutions. Gérard Mégie suivait de près les méandres, parfois bien déroutants pour le scientifique, des négociations sur les CFC; il y était même directement impliqué en tant que scientifique. Il avait rapidement compris que la question de l'articulation entre l'apport de connaissances des scientifiques et la décision sociale ou politique était cruciale. Il y voyait un problème posé à l'activité, censée être socialement utile, qu' est la recherche. Il ressentait de ce fait fortement le besoin pour celle-ci de réexaminer ses pratiques pour qu'elle puisse faire face aux enjeux contemporains d'une action fondée sur la connaissance. Il avait tiré de cette expérience une vraie curiosité pour les sciences de la société, découvrant ainsi que le fonctionnement des sociétés et l'action politique s'analysent, eux aussi, scientifiquement. Le rapport sciences/action devenait pour lui un enjeu de connaissance en soi.

Cela rencontrait - et c'est là la seconde origine de son cheminement vers l'interdisciplinarité - sa conception civique du rôle du chercheur; plus, cela la confortait. Que les scientifiques aient une responsabilité propre à assumer vis-à-vis de la société était en effet, chez lui, une 
conviction particulièrement forte, qui se traduisait par de multiples engagements, comme le montrèrent plusieurs des témoignages. Ces engagements allaient du souci de s'impliquer, par son apport d'expertise, dans les réflexions sur des applications des connaissances mettant en cause l'environnement, à l'engagement politique, en passant par une action soutenue, constante, militante même, en faveur de l'éthique scientifique. Autant d'occasions pour lui de mieux prendre la mesure des dimensions sociales de son activité de chercheur et des implications sociétales des connaissances scientifiques sur lesquelles elle débouchait. Il en est donc très tôt venu à considérer qu'une démarche pluridisciplinaire incluant l'apport des sciences sociales était indispensable pour traiter de la question de l'ozone, mais aussi, plus généralement, de toutes les questions environnementales.

Mais il restait à passer de ce simple constat à la conceptualisation d'une démarche de recherche répondant à cette exigence. C'est là qu'entre en ligne de compte la troisième source de son adhésion à l'interdisciplinarité. Cette troisième source, ce sont ses qualités personnelles : sa soif de comprendre, sa capacité d'embrasser large, sa curiosité, son intérêt porté à l'autre, sa confiance dans la démarche scientifique, son intelligence ennemie de toutes les œillères. Ce sont ces qualités qui l'ont conduit à dépasser l'idée de la simple juxtaposition pluridisciplinaire pour chercher la voie de l'intégration des disciplines dans une démarche commune.

Sur la question de l'interdisciplinarité, sa pensée a sans cesse été en mouvement depuis la publication de son ouvrage Ozone, l'équilibre rompu aux Presses $\mathrm{du}$ CNRS en 1989. Le point d'aboutissement de cette réflexion est contenu dans le projet d'établissement du CNRS de février $2002^{1}$. L'inspiration qui l'a guidé dans la mise au point de ce projet était en écho complet de ce qu'il attendait de la revue Natures Sciences Sociétés et de ce pour quoi il avait accepté d'en être un des rédacteurs en chef fondateurs : 1 'établissement des bases d'une interdisciplinarité réelle, théoriquement fondée, rigoureuse parce qu'évaluable et évaluée. Il est significatif qu'il ait fait appel à l'un de ses corédacteurs en chef (Jean-Marie Legay) pour animer le groupe de travail chargé de préparer ce projet par une réflexion sur l'analyse des systèmes complexes. Jean-Marie Legay a pu dire, lors de son intervention dans ces journées, que ce choix lui semblait lié au travail accompli ensemble pendant douze années au sein de la revue.

Les événements ont voulu que ce texte, malheureusement, devienne une sorte de testament. Mais ce n'est pas ainsi, bien sûr, qu'il convient de le lire. Car ce qu'à travers lui, Gérard Mégie voulait dire, c'est que, pour être de plain-pied dans le XXI ${ }^{\mathrm{e}}$ siècle, la recherche ne pourra pas faire l'économie des questions qu'il soulève. Le meilleur hommage que l'on puisse rendre à son initiateur est de s'approprier ce texte trop méconnu et pourtant totalement tourné vers l'avenir.

Les Journées se voulaient «sur les traces d'un scientifique humaniste ». Curieusement, la traduction anglaise voyait, quant à elle, Gérard Mégie comme un «visionary scientist ». Ce n'est pas le même regard qui est porté : on honore l'humaniste pour ce qu'il a été, on s'inspire du visionnaire pour la voie qu'il trace. Que l'Académie des sciences crée une fondation et un prix Gérard Mégie, que le siège social du CNRS devienne le «Campus Gérard Mégie », que tel ou tel amphithéâtre prenne ce nom, toutes décisions annoncées lors des Journées, on ne peut que s'en réjouir! Mais craignons le coup de grâce du coup de chapeau! Le hasard a voulu que ces Journées se tiennent au moment où le CNRS traverse une grave crise marquée par des changements de personnes à sa tête même. Un héritage est à défendre : celui d'un scientifique "humaniste» et "visionnaire». Et visionnaire précisément parce qu'humaniste. L'humaniste a été honoré. Maintenant, suivons le visionnaire!

Marcel Jollivet

${ }^{1}$ Cf. le compte rendu de ce document dans Natures Sciences Sociétés, 11, 1 (2003), 71-78. 